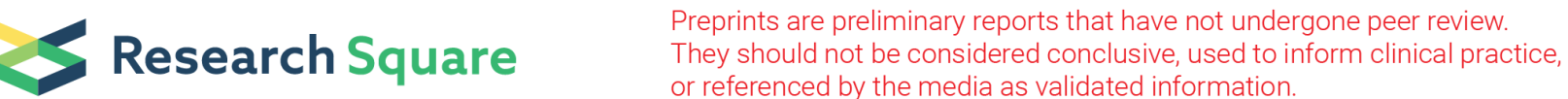

\section{Synthesis of p-n CoO-ZnO Heterojunction for Enhanced Visible-Light Assisted Photodegradation of Methylene Blue}

\section{Muhammad Saeed ( $\sim$ msaeed@gcuf.edu.pk)}

Government College University Faisalabad https://orcid.org/0000-0002-8759-6948

\section{Muhammad Adeel}

Government College University Faisalabad

Iltaf Khan

Beijing Institute of Petrochemical Technology

Nadia Akram

Government College University Faisalabad

Majid Muneer

Government College University Faisalabad

\section{Research Article}

Keywords: Photodegradation, p-n heterojunction, CoO-ZnO, Eley-Rideal mechanism

Posted Date: July 30th, 2021

DOl: https://doi.org/10.21203/rs.3.rs-703284/v1

License: (c) (i) This work is licensed under a Creative Commons Attribution 4.0 International License. Read Full License 
1 Synthesis of p-n $\mathrm{CoO}-\mathrm{ZnO}$ heterojunction for enhanced visible-light assisted

2 photodegradation of methylene blue

3 Muhammad Saeed $^{* 1}$, Muhammad Adeel ${ }^{1}$, Iltaf Khan ${ }^{2}$, Nadia Akram ${ }^{1}$, Majid Muneer ${ }^{1}$

$4 \quad{ }^{1}$ Department of Chemistry, Government College University Faisalabad Pakistan

$5 \quad{ }^{2}$ College of Chemical and Materials Engineering, Beijing Institute of Petrochemical Technology, 6 Beijing, P. R. China

7 ABSTRACT

8 The synthesis of p-n heterojunction has received much attention in the eradication of organic

9 pollutants from water. In this study, $\mathrm{p}-\mathrm{n} \mathrm{CoO}-\mathrm{ZnO}$ heterojunction is reported as an efficient

10 visible-light-driven photocatalyst for degradation of methylene blue. The formation of $\mathrm{CoO}-\mathrm{ZnO}$

11 was determined by XRD, XPS, UV-vis DRS, SEM, and EDS analyses. The p-n CoO-ZnO

12 prepared by precipitation method showed an enhanced photocatalytic activity under the

13 irradiation of visible light compared to $\mathrm{ZnO}$. A $100 \mathrm{mg} / \mathrm{L}$ methylene blue is degraded almost

14 completely within 2 hours under visible irradiation. The enhanced photocatalytic efficiency is

15 attributed to the synergistic effect of $p$-n heterojunction that dramatically reduces the

16 recombination of holes and electrons initiated by irradiation. The role played by hydroxyl

17 radicals in photodegradation of methylene blue was determined by photoluminescence emission 18 and fluorescence spectroscopy.

20 Keywords: Photodegradation, p-n heterojunction, $\mathrm{CoO}-\mathrm{ZnO}$, Eley-Rideal mechanism

*msaeed@gcuf.edu.pk 


\section{INTRODUCTION}

22 The wastewater of industries such as textile, plastic, pharmaceutical, printing, leather, cosmetics,

23 food processing, and dye manufacturing contains several kinds of synthetic dyes. Due to high

24 stability and non-biodegradable nature, these dyes pose not only toxicity to aquatic life but also

25 ecological risks (Zhang and Camarena 2020) (Katheresan et al. 2018) (Pavithra et al. 2019).

26 Therefore, it is very important to remove these dyes from industrial effluent before their release to

27 the aqueous system. Various strategies such as adsorption (Ma et al. 2019), photocatalytic

28 degradation (Menazea et al. 2020) (Abdelghany et al. 2020), membrane filtration (Lu et al. 2019),

29 coagulation (Wang et al. 2017), chemical oxidation (Hayat et al. 2015) and biological degradation

30 (Kanagaraj et al. 2015) have been used for the eradication of dyes from industrial effluents. Due

31 to low cost, simplicity, and high efficiency, photocatalytic degradation using semiconductor metal

32 oxides as catalysts is the recommended technique for the treatment of dyes contaminated industrial

33 effluents. Complete cycles of photocatalytic degradation comprise of several steps including (1)

34 the harvesting of light, (2) the generation of the exciton (pair of electron and hole), (3) the

35 generation of $\mathrm{OH}$ radicals by secondary reactions of the exciton, and (4) mineralization of organic

36 pollutants by reactions with OH radicals (Saeed et al. 2020) (Nisar et al. 2020) (Noruozi and

37 Nezamzadeh-Ejhieh 2020). However, the fast recombination of electron and hole affects the

38 efficiency of the aforementioned processes and finally results in a drastic decrease in

39 photocatalytic activity towards the photodegradation of organic pollutants. This fast recombination

40 of photoinduced electron and hole is the most important negative aspect of semiconductor metal

41 oxides photocatalysts. Several techniques including metal or non-metal doping, the formation of

42 plasmonic structures, supporting the semiconductor metal oxides onto suitable supports such as 
43 zeolites, and formation of heterojunction by coupling of two or more semiconductors have been

44 used for preventing/slowing down the recombination of photoinduced electron and hole.

45 Herein, we report the coupling of cobalt oxide with zinc oxide to get an efficient p-n CoO-ZnO 46 heterojunction as photocatalyst for visible light assisted degradation of methylene blue. $\mathrm{ZnO}$ is an

47 n-type semiconductor characterized by environment-friendly nature, low cost, conductivity in 48 electronics, and stability. Unfortunately, it can't be used as a photocatalyst for the degradation of 49 organic pollutants under visible light irradiation due to its hard response to visible photons (Fu et 50 al. 2016). The $\mathrm{CoO}-\mathrm{ZnO}$ can be used as an efficient visible-light-driven photocatalyst because the 51 construction of $\mathrm{p}-\mathrm{n}$ heterojunction effectively facilitates the formation of electron-hole pair and its 52 separation. Cobalt oxide is a p-type semiconductor characterized by band gaps of 1.48 and 2.19 $53 \mathrm{eV}$. The p-n $\mathrm{CoO}-\mathrm{ZnO}$ heterojunction formed by the coupling of cobalt oxide and zinc oxide leads 54 to the formation of an inner electric field (Mohamed Reda et al. 2017). As a result, the visible 55 light-harvesting ability of $\mathrm{CoO}-\mathrm{ZnO}$ improves and ultimately the photocatalytic activity of $\mathrm{p}-\mathrm{n}$ $56 \mathrm{CoO}-\mathrm{ZnO}$ towards the degradation of organic pollutants is enhanced.

\section{2. EXPERIMENTAL}

\section{$58 \quad 2.1$ Synthesis of CoO-ZnO}

$\mathrm{CoO}-\mathrm{ZnO}$ was prepared by a chemical precipitation method using $2 \mathrm{M} \mathrm{NaOH}$ solution as a

60 precipitating agent. Typically, a $0.4 \mathrm{M}(50 \mathrm{~mL})$ solution of zinc acetate dihydrate was mixed

61 with $0.1 \mathrm{M}(30 \mathrm{~mL})$ solution of cobalt nitrate hexahydrate for the synthesis of $\mathrm{CoO}-\mathrm{ZnO}$ loaded

62 with $10 \%$ Co. Then, 2 M sodium hydroxide solution was added dropwise to a mixed solution of

63 zinc acetate dihydrate and cobalt nitrate hexahydrate under continuous stirring until pH 10 was

64 obtained. The resultant reaction mixture was stirred further for two hours for the completion of 
65 the reaction. Afterward, the resultant precipitate was filtered and dried at $100{ }^{\circ} \mathrm{C}$ for 12 hours.

66 Finally, the obtained solid was calcined at $450{ }^{\circ} \mathrm{C}$ for 4 hours. Similarly, CoO-ZnO loaded with

$675 \%$, and $15 \%$ Co were prepared for comparison.

68

69

70

71

72

73

74

75

76

77

78

79

80

81

82 activity.

83 3. RESULTS AND DISCUSSION

84

\subsection{Characterization}

\subsection{Photocatalytic experiment}

3.1 Characterization

X-Ray Diffraction (XRD), X-ray photoelectron spectroscopy (XPS), Diffuse Reflectance UVvisible (DR-UV-Vis) spectroscopy, Scanning electron microscopy (SEM) and Energy dispersive spectroscopy (EDS) were used for characterization of prepared material.

To evaluate the photocatalytic activities of as-prepared CoO-ZnO, a $500 \mathrm{~mL}$ Pyrex glass beaker, as a batch reactor, was charged with a $50 \mathrm{~mL}$ solution of methylene blue as a model pollutant. Then, an appropriate amount of $\mathrm{CoO}-\mathrm{ZnO}$ was added as a photocatalyst to the methylene blue solution. The resultant suspension was stirred for half an hour to get the adsorption-desorption equilibrium. After 30 minutes of stirring under dark, a $0.5 \mathrm{~mL}$ sample was pipette-out and analyzed with a UV-visible spectrophotometer for any change in concentration due to stirring under dark condition. Then, the suspension was irradiated with visible light under continuous stirring. About $0.5 \mathrm{~mL}$ of suspension was sampled at a time interval of 30 minutes. The sample suspensions were analyzed with a UV-visible spectrophotometer to estimate the photocatalytic 
85 The XRD pattern given in Figure 1a is dominated with sharp peaks which indicate the crystalline

86 nature of as prepared $\mathrm{CoO}-\mathrm{ZnO}$. The diffraction peaks at $2 \theta$ degrees $76.99,72.59,68.11,67.97$,

$8762.88,56.62,47.55,36.27$ and 34.43 represent the (202), (004), (201), (112), (103), (110), (102),

88 (101) and (002) planes of $\mathrm{ZnO}$ respectively (JCPDS card no. 89-0510) (Adeleke, J. T.,

89 Theivasanthi, T., Thiruppathi, M., Swaminathan, M., Akomolafe, T., \& Alabi 2018) (Kang, K.

90 M., Wang, Y., Kim, M., \& Park 2018). Cobalt oxide gives a representative diffraction peak at $2 \theta$

$91 \sim 36^{\circ}$ (Saeed et al. 2018). The magnified diffraction peak at $2 \theta 36.27$ in XRD of CoO-ZnO

92 shows that it has been shifted by 0.12 compared to $\mathrm{ZnO}$. This shift in peak position shows that

$93 \mathrm{Co}^{2+}$ has been doped in $\mathrm{ZnO}$ (Yang et al. 2019).

94 The elemental and chemical states of prepared $\mathrm{CoO}-\mathrm{ZnO}$ were determined by XPS analysis. The $95 \mathrm{Zn} 2 \mathrm{p}$ core-level spectra given in Figure $1 \mathrm{~b}$ show the existence of $\mathrm{Zn} 2 \mathrm{p} 3 / 2$ and $\mathrm{Zn} 2 \mathrm{p} 1 / 2$ regarding 96 peaks at binding energy $\sim 1022 \mathrm{eV}$ and $\sim 1047 \mathrm{eV}$ respectively (Reddy et al. 2020) (Achouri et al. 97 2016). The O 1s core-level spectra given in Figure 1c consist of two peaks at binding energy 529.3 $98 \mathrm{eV}$ due to lattice oxygen $\left(\mathrm{O}_{\mathrm{L}}\right)$ and $531.5 \mathrm{eV}$ due to chemisorbed oxygen $\left(\mathrm{O}_{\mathrm{H}}\right)$. These peaks are 99 attributed to intrinsic $\mathrm{O}^{2-}$ ions in the wurtzite structure of the hexagonal $\mathrm{Zn}^{2+}$ ion array and $\mathrm{O}^{2-}$ 100 ions in the oxygen-deficient area of $\mathrm{ZnO}$ respectively (Zhang et al. 2011) (G.H. Kim, D.L. Kim, 101 B.D. Ahn, S.Y. Lee 2009). Similarly, the Co 2p spectrum of CoO-ZnO given in Figure 1d shows 102 peaks at $780.3 \mathrm{eV}$ and $795.8 \mathrm{eV}$ attributed to $\mathrm{Co} 2 \mathrm{p} 3 / 2$ and $\mathrm{Co} 2 \mathrm{p} 1 / 2$ respectively. The difference 103 in the binding energy of these two peaks $(15.5 \mathrm{eV})$ matches standard CoO. Based on the results 104 of XRD and XPS, it is confirmed that Co exists in a divalent state in as-prepared composite (Wang 105 et al. 2012) (Cao et al. 2009). Hence, we report that the as-prepared sample is CoO-ZnO.

106 The UV-visible DRS spectra of $\mathrm{ZnO}$ and $\mathrm{CoO}-\mathrm{ZnO}$ given in Figure 1e shows that the implantation 107 of $\mathrm{Co}$ shifts the light absorption capability of $\mathrm{CoO}-\mathrm{ZnO}$ to the longer wavelength side. The 
108 estimated band gaps for $\mathrm{ZnO}$ and $\mathrm{CoO}-\mathrm{ZnO}$ are $3.37 \mathrm{eV}$ and $2.16 \mathrm{eV}$ respectively which show that 109 implantation of $\mathrm{Co}$ in $\mathrm{ZnO}$ structure narrows the band gap. Hence, $\mathrm{CoO}-\mathrm{ZnO}$ can be employed as 110 an effective visible light photocatalyst for the degradation of organic pollutants (Chauhan et al. 111 2019) (Mohamed et al. 2016) (Kumar et al. 2016).
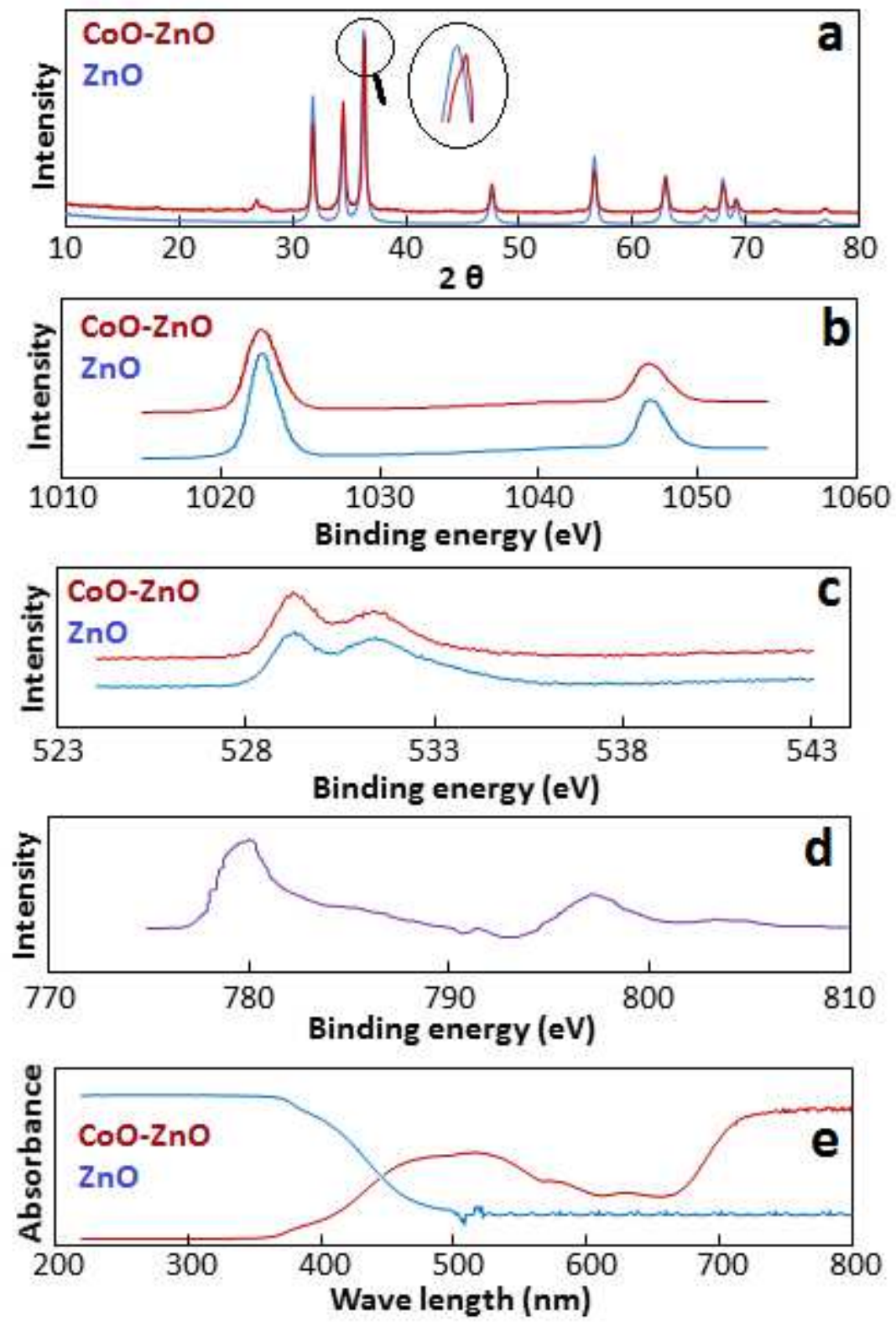
113 Figure 1 Characterization of catalysts a) XRD pattern, b) XPS Spectra of Zn 2p, c) XPS O 1s

114 core-level spectra, d) XPS Co 2p spectrum of CoO-ZnO, e)DRS UV/Vis spectra of ZnO and $115 \mathrm{CoO}-\mathrm{ZnO}$

117 The morphology of as-prepared $\mathrm{CoO}-\mathrm{ZnO}$ was investigated by SEM analysis. The SEM 118 micrograph given in Figure $2 \mathrm{~b}$ shows that the $\mathrm{CoO}-\mathrm{ZnO}$ is composed of nanosheets of various 119 diameters. The SEM micrograph shows that the particles are non-agglomerated and dispersed. 120 The comparison of SEM micrographs of $\mathrm{ZnO}$ and $\mathrm{CoO}-\mathrm{ZnO}$ revealed that the implantation of Co 121 in $\mathrm{ZnO}$ caused an increase in the diameter of $\mathrm{CoO}-\mathrm{ZnO}$. During the synthesis of $\mathrm{CoO}-\mathrm{ZnO}$ 122 heterojunction, the reaction of $\mathrm{Co}^{2+}$ with hydroxide reduces the reaction of $\mathrm{Zn}^{2+}$ with hydroxide 123 which affects the overall nucleation process. As a result of this mechanism, the lateral growth of 124 the particles is promoted and ultimately, the diameter of the particle is increased (Kaphle et al. 125 2019) (Kaphle et al. 2018). The as-prepared $\mathrm{CoO}-\mathrm{ZnO}$ was further characterized by EDS 126 analysis. EDS analysis showed that as prepared $\mathrm{CoO}-\mathrm{ZnO}$ is composed of $\mathrm{Co}, \mathrm{Zn}$, and $\mathrm{O}$ only. 127 The atomic percentage of $\mathrm{Co} \mathrm{Zn}$ and $\mathrm{O}$ was found as $2.41 \%, 31.93 \%$, and $65.67 \%$ respectively. 128 The EDS analysis also revealed that the elements Co, Zn, and O were homogeneously distributed 129 as given in Figures 2c-2f). 

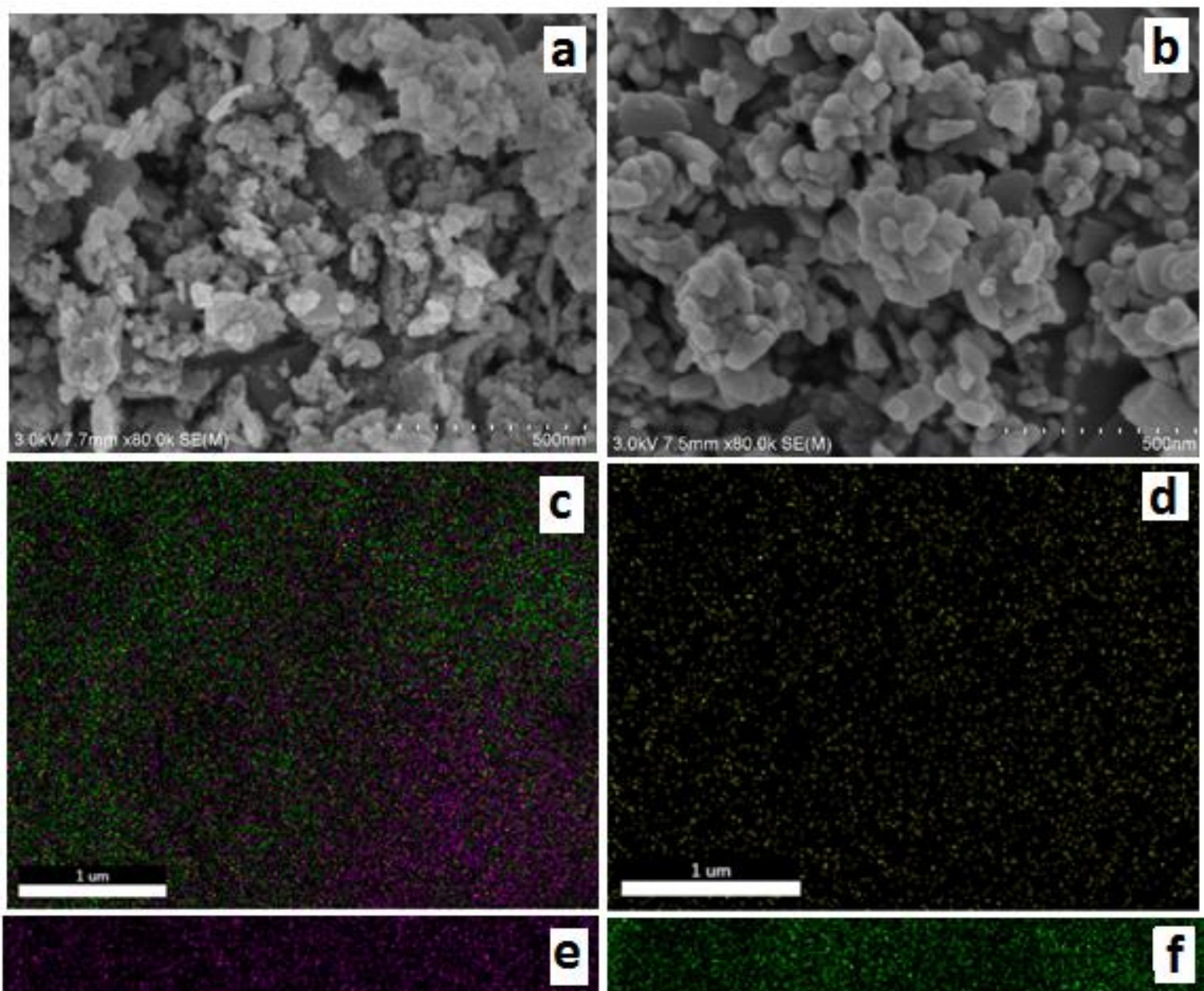

131 Figure 2 a) SEM micrograph of $\mathrm{ZnO}$, b) SEM micrograph of $\mathrm{CoO}-\mathrm{ZnO}$, c) Elemental mapping 132 of CoO-ZnO, d) Distribution of Co, s) Distribution of $\mathrm{Zn}, \mathrm{f}$ ) Distribution of O 
135

136

137

138

139

140

141

142

143

144

145

146

147

148

149

150

151

152

\subsection{Photocatalytic activity}

Figure 3 shows the photocatalytic activity of as-prepared photocatalysts towards

photodegradation of methylene blue. For comparison of photocatalytic activities of pure $\mathrm{ZnO}$ and $\mathrm{CoO}-\mathrm{ZnO}$ heterojunctions, photodegradation experiments were performed by irradiation of $100 \mathrm{mg} / \mathrm{L}$ solution $(50 \mathrm{~mL})$ of methylene blue and $0.05 \mathrm{~g}$ of the catalyst under visible light at 30 ${ }^{\circ} \mathrm{C}$ for 120 minutes. Before irradiation, the catalyst and methylene blue solution were magnetically stirred for 30 minutes under the dark condition to get adsorption-desorption equilibrium. Similarly, a blank experiment was also conducted by stirring the pristine dye solution (without catalyst) for one hour. The $\mathrm{CoO}-\mathrm{ZnO}$ samples exhibited higher photocatalytic activity than pure $\mathrm{ZnO}$ which shows that the implantation of $\mathrm{Co}$ in $\mathrm{ZnO}$ enhances the photocatalytic activity. However, the increasing content of Co leads to a decrease in photocatalytic activity under the same experimental conditions. The decrease in photocatalytic activity with higher Co content is attributed to the agglomeration of Co in heterojunction. Analyses of reaction mixtures showed about 9, 64, 96, and 79\% removal of methylene blue in the presence of pure $\mathrm{ZnO}, 5 \% \mathrm{CoO}-\mathrm{ZnO}, 10 \% \mathrm{CoO}-\mathrm{ZnO}$, and $15 \% \mathrm{CoO}-\mathrm{ZnO}$ respectively. Due to the high band gap $(3.37 \mathrm{eV})$, the formation of exciton cannot be initiated by visible irradiation of $\mathrm{ZnO}$, therefore $\mathrm{ZnO}$ exhibited lower photocatalytic activity. The removal of methylene blue in the presence of $\mathrm{ZnO}$ is attributed to the photosensitization of methylene blue. The $10 \% \mathrm{CoO}-$ $\mathrm{ZnO}$ exhibited the highest photocatalytic activity in this study. The effectiveness of catalysts is expressed by using the plot of $\mathrm{MB}_{\mathrm{t}} / \mathrm{MB}_{\mathrm{o}}$ vs time as given in Figure $3 \mathrm{a}\left(\mathrm{MB}_{\mathrm{o}}\right.$ : initial concentration, $\mathrm{MB}_{\mathrm{t}}$ : final concentration, $\mathrm{t}$ : degradation time).

As $\mathrm{pH}$ of reaction mixture significantly affects the photocatalytic efficiency, therefore the $\mathrm{pH}$ dependence of visible light assisted photodegradation of methylene blue in the presence of $10 \%$ 
$158 \mathrm{CoO}-\mathrm{ZnO}$ photocatalyst was investigated. A reaction mixture composed of $100 \mathrm{mg} / \mathrm{L}(50 \mathrm{~mL})$ 159 solution of methylene blue and $0.05 \mathrm{~g}$ of the photocatalyst was magnetically stirred under visible 160 irradiation over a $\mathrm{pH}$ range of 2-10. Analysis of reaction mixture showed that photodegradation 161 efficiency increases with an increase in $\mathrm{pH}$ as given in Figure $3 \mathrm{~b}$. The enhanced photocatalytic 162 activity at higher $\mathrm{pH}$ is explained based on point of zero charge (PZC) of $\mathrm{ZnO}$; $\mathrm{pH}$ at which the 163 fraction of positively charged and negatively charged surface becomes equal. The PZC of $\mathrm{ZnO}$ is 164 9. At $\mathrm{pH}$ greater than 9 , the surface of $\mathrm{ZnO}$ is negatively charged, which favors the formation of $165 \mathrm{OH}$ radicals. It results in enhanced photocatalytic activity at $\mathrm{pH} 10$. On the other hand, lower $\mathrm{pH}$ 166 causes a decrease in photocatalytic activity due to electrostatic repulsion between positively 167 charged $\mathrm{ZnO}$ and cationic dye (Prabakaran and Pillay 2019) (Vuppala et al. 2012). Also, the 168 optimum catalyst dose was investigated by performing photodegradation experiments with 169 catalyst dose $0.01,0.05,0.1$, and $0.15 \mathrm{~g}$ separately. The catalyst dose $0.05 \mathrm{~g}$ was found as the 170 optimum catalyst dose. Furthermore, a good catalyst is one which can be recycled many times 171 therefore the cycling stability of $10 \% \mathrm{CoO}-\mathrm{ZnO}$ was also evaluated. The photodegradation 172 experiment was carried out by suspending $0.05 \mathrm{~g}$ of $\mathrm{CoO}-\mathrm{ZnO}$ in $50 \mathrm{~mL}$ methylene blue solution, 173 after 60 minutes of stirring the suspension under irradiation of visible light, the $\mathrm{CoO}-\mathrm{ZnO}$ was 174 separated, washed, and dried. Then, the dried residue was dispersed in new solution of methylene 175 blue. It was found that $\mathrm{CoO}-\mathrm{ZnO}$ exhibited almost same catalytic activity after 3 consecutive 176 cycles.

177 Similarly, the effect of the initial concentration of methylene blue on photocatalytic efficiency 178 was also studied. Figure $3 c$ shows that catalytic efficiency decreases with an increase in the 179 concentration of methylene blue. The decrease in catalytic efficiency with a higher concentration 180 of dye is due to (1) dye absorbs a significant fraction of light rather than a catalyst, (2) the path 
181 length of a photon decreases with an increase in the concentration of dye, (3) the ration of $\mathrm{OH}$

182 radicals to molecules of dye decreases with increase in the concentration of dye (Balcha et al.

183 2016) (Saeed et al. 2016).
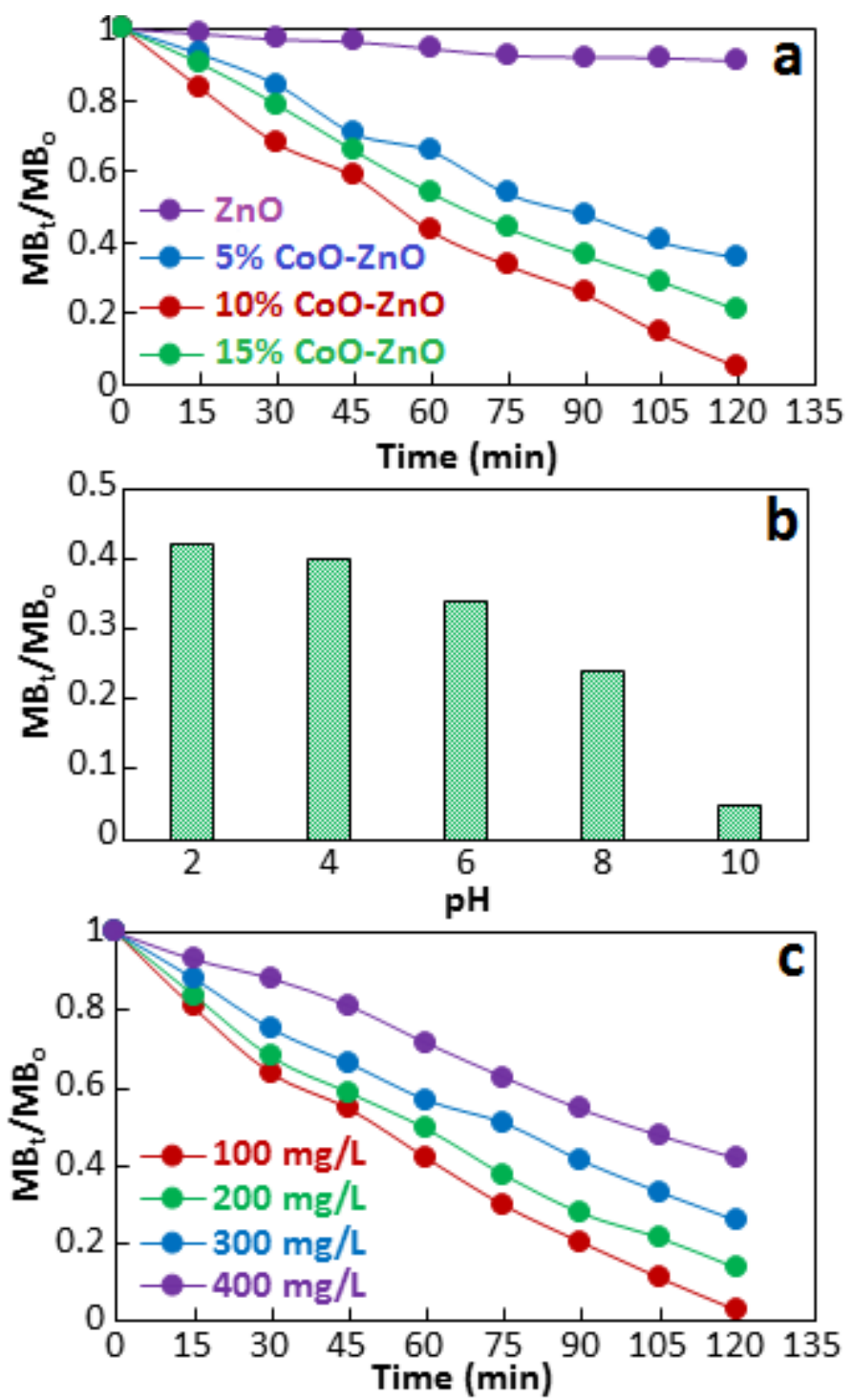

Figure 3 Photodegradation of methylene blue under visible irradiation a) Comparison of

186 photocatalytic activities of different catalysts, b) pH dependence of $10 \% \mathrm{CoO}-\mathrm{ZnO}$ catalyzed

187 photodegradation of methylene blue, c) Concentration dependence of $10 \% \mathrm{CoO}-\mathrm{ZnO}$ catalyzed

188 photodegradation of methylene blue 


\subsection{Photocatalytic mechanism and kinetics}

191 Generally, it is well-known that the irradiation of photocatalyst generates electrons and holes and

192 then move to the surface of photocatalyst. These photo-initiated electrons and holes participate in

193 a series of redox reactions and give hydroxyl radicals that take part in the degradation of organic

194 pollutants. Therefore, the reactive species trapping experiments were carried out to insight the

195 mechanism. Isopropyl alcohol and ammonium oxalate were used as a scavenger for hydroxyl

196 radicals and holes, respectively (Liu and Li 2018). It was found that the addition of isopropyl

197 alcohol and ammonium oxalate decreased the photocatalytic activity from $95 \%$ to $63 \%$ and $71 \%$

198 respectively. It confirmed that hydroxyl radicals and holes were the imperative species during

199 photodegradation of methylene blue dye. Based on obtained results, a possible mechanism is put

200 forward for the photodegradation of methylene blue over $\mathrm{CoO}-\mathrm{ZnO}$ (Figure 4a). The higher

201 photocatalytic activity of $\mathrm{CoO}-\mathrm{ZnO}$ is due to the synergetic effect of cobalt oxide and zinc oxide.

202 At the interface of $\mathrm{p}-\mathrm{n} \mathrm{CoO}-\mathrm{ZnO}$ heterojunction, the electrons transfer from $\mathrm{p}$ to $\mathrm{n}$ until their

203 Fermi levels get equilibrium and it gives rise to an internal electric field (Dong et al. 2015).

204 When $\mathrm{CoO}-\mathrm{ZnO}$ heterojunction is irradiated, the electrons get excited from $\mathrm{VB}$ to $\mathrm{CB}$ in both

205 cobalt oxide and zinc oxide. The internal electric field drives the photoinduced electrons from

206 CB of p-type cobalt oxide to CB of n-type zinc oxide. At the same time, the holes move from VB

207 of n-type zinc oxide to VB of p-type cobalt oxide, thus the rate of recombination of holes and

208 electrons is reduced resulting in enhanced photocatalytic activity (Zhang et al. 2020). The

209 separation efficiency of photoinduced electrons and hole was further verified by

210 photoluminescence emission spectra (PL) of $\mathrm{ZnO}$ and $\mathrm{CoO}-\mathrm{ZnO}$ samples. Figure $4 \mathrm{~b}$ shows that

211 PL intensity of $\mathrm{CoO}-\mathrm{ZnO}$ is lower than $\mathrm{ZnO}$ which indicates that band gap of $\mathrm{CoO}-\mathrm{ZnO}$ is lower 
212 than $\mathrm{ZnO}$. The lower PL intensity shows that the formation of $\mathrm{p}-\mathrm{n} \mathrm{CoO}-\mathrm{ZnO}$ heterojunction

213 reduces the rate of recombination of electrons-holes, initiates the charge transfer, and ultimately

214 enhances the photocatalytic activity. The proposed mechanism was further confirmed by

215 Fluorescence spectroscopy related to hydroxyl radical measurements as given in Figure 4c. The

216 higher fluorescent intensity of $\mathrm{CoO}-\mathrm{ZnO}$ shows that $\mathrm{OH}$ radicals are more radially produced in

217 the presence of $\mathrm{CoO}-\mathrm{ZnO}$ leading to enhanced photocatalytic activity (Alamdari et al. 2019)

218 (Bhakta and Chakrabarti 2019) (Kang et al. 2018) (Khan et al. 2020) (Khan et al. 2019). 

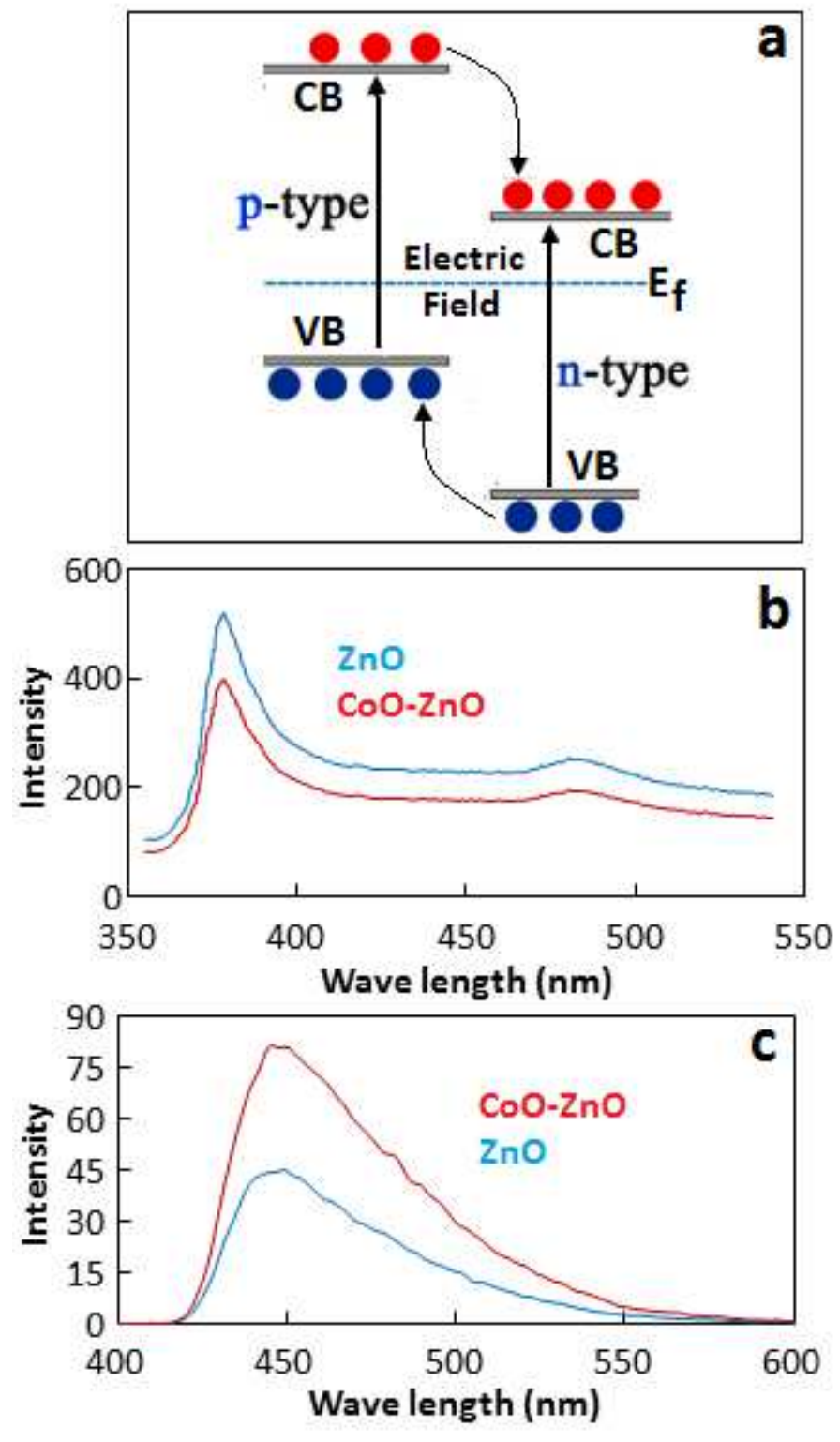

219

220 Figure 4 a) Mechanism of photocatalytic degradation, b) Photoluminescence (PL) emission

221 spectra, c) Fluorescence spectra related to hydroxyl radical measurements

222 The reaction mechanism discussed above is outlined below.

$223 \mathrm{CoZnO}_{2}+$ Irradiation $\rightarrow \mathrm{CB}\left(h^{+}\right)+\mathrm{VB}\left(e^{-}\right)$

$224 \mathrm{CB}\left(h^{+}\right)+\mathrm{H}_{2} \mathrm{O} \rightarrow \mathrm{OH}$ 
$225 V B\left(e^{-}\right)+\left(O_{2}\right)_{\text {adsorbed }} \rightarrow \mathrm{O}_{2}^{-}$

$226 \mathrm{O}_{2}^{-}+\mathrm{H}_{2} \mathrm{O} \rightarrow \mathrm{OH}$ (4)

$227 \quad O H+M B \rightarrow$ Degradation products

228 Based on above reactions, the rate photocatalytic degradation is written as $\left(\mathrm{k}_{\mathrm{r}}\right.$ is rate constant of 229 photodegradation)

$230-\frac{d M B}{d t}=k_{r}$ Irradiation $\left(\mathrm{O}_{2}\right)_{\text {adsorbed }} M B$

231 Rate expression 6 represents a typical Eley-Rideal type mechanism of heterogeneous catalytic

232 reactions. As the reaction mixture is irradiated continuously and is open to the atmosphere,

233 therefore, the rate of reaction becomes independent of irradiation and oxygen. Hence, rate

234 expression becomes as

$235-\frac{d M B}{d t}=k_{r} M B$

236 On integration, the rate expression becomes as

$237 \ln \frac{M B_{O}}{M B_{t}}=k_{r} t$

238 The degradation vs time data with various initial concentrations of methylene blue was analyzed

239 according to rate expression 8 as given in Figure 4a. The rate constant, $\mathrm{k}_{\mathrm{r}}$, were $0.0179,0.0138$,

$240 \quad 0.0098$ and 0.0064 per minute with initial concentration $100,200,300$ and $400 \mathrm{mg} / \mathrm{L}$

241 respectively. It can be observed that the rate constant decreased with an increase in the initial

242 concentration of methylene blue. As explained in the previous section, the rate constant

243 decreased with increased concentration due to (1) dye absorbs a significant fraction of light

244 rather than a catalyst, (2) the path length of a photon decreases with an increase in the 
245 concentration of dye, (3) the ration of $\mathrm{OH}$ radicals to molecules of dye decreases with increase in

246 the concentration of dye. Similarly, the degradation vs time data at different temperatures was

247 also analyzed as given in Figure 5b. The rate constant, $\mathrm{k}_{\mathrm{r}}$, were $0.0158,0.0179$ and 0.0188 per

248 minute for photodegradation reaction carried out at 30,40 , and $50{ }^{\circ} \mathrm{C}$, respectively. The rate of a

249 photochemical reaction does not depend on temperature, but we observed a slight increase in the

250 rate constant with an increase in temperature. The increase in the rate constant with temperature

251 is due to an increase in the kinetic energy of molecules at a higher temperature.
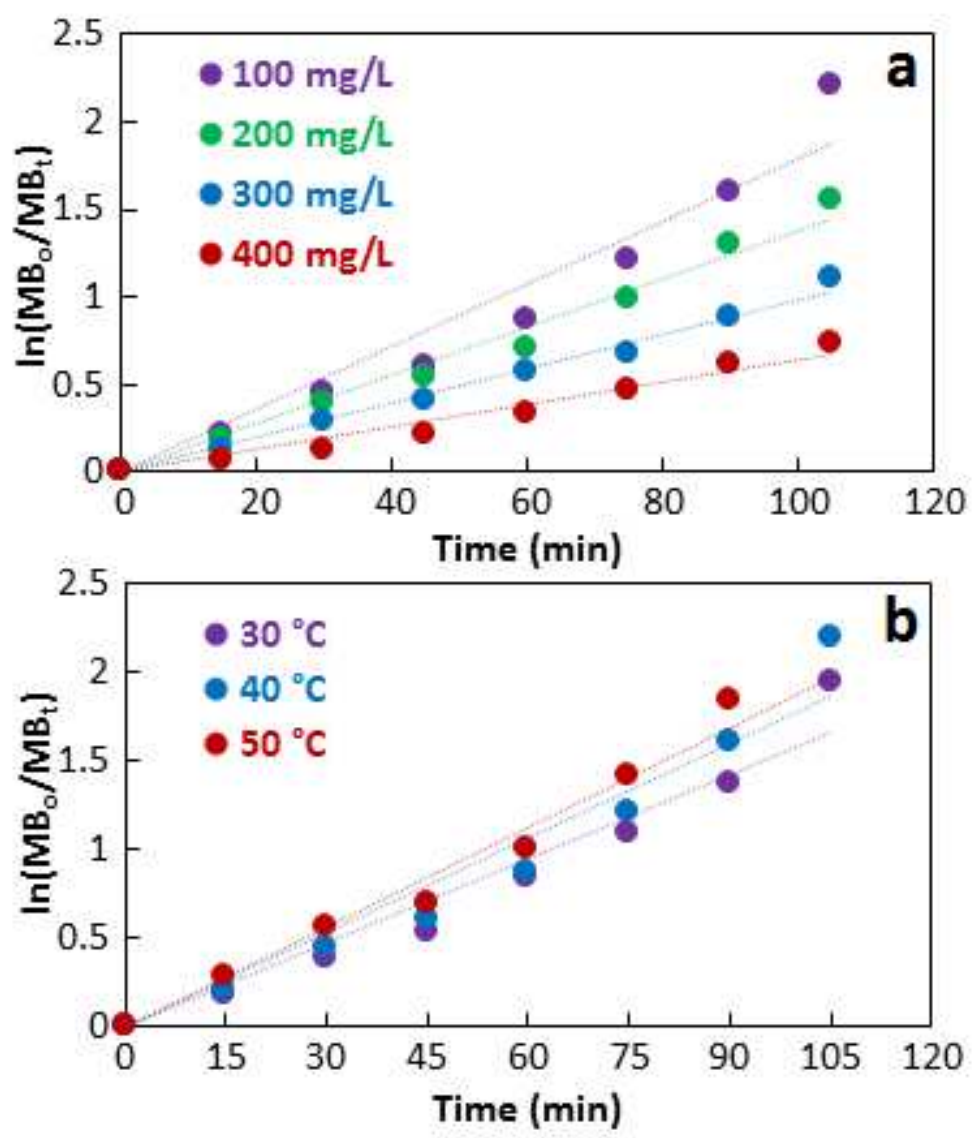

252

253 Figure 5 Kinetics analyses of degradation vs time data a) with various initial concentration of

254 methylene blue, b) at different temperatures

\section{Conclusions}


256 p-n CoO-ZnO heterojunction was synthesized successfully by precipitation method and

257 characterized by XRD, XPS, UV-vis, SEM, and EDS analyses. The as-prepared heterojunction

258 was successfully employed as photocatalyst for enhanced photodegradation of methylene blue

259 under irradiation of visible light. A $100 \mathrm{mg} / \mathrm{L}$ solution of methylene blue $(50 \mathrm{~mL})$ was almost

260 completely degraded within 2 hours under visible irradiation over $0.05 \mathrm{~g}$ CoO-ZnO photocatalyst.

261 The higher photocatalytic activity of $\mathrm{CoO}-\mathrm{ZnO}$ is due to the synergetic effect of cobalt oxide and

262 zinc oxide. The internal electric field formed at the interface of heterojunction boosted the

263 separation of photoinitiated holes and electrons and thus resulted in increased photocatalytic

264 activity. The reaction kinetics was described in terms of Eley-Rideal mechanism.

265 Declarations

266 Ethics approval and consent to participate

267 Not applicable

268 Competing Interests

269 There is no competing interest with this manuscript.

270 Consent for publication

271 Not applicable

272 Authors Contributions

273 Muhammad Saeed is main research supervisor, who has supervised the experimental work and

274 drfted the manuscript.

275 Muhammad Adeel is research student who has carried out the experimental work. 
276 Iltaf Khan has provided the characterization fascilities.

277 Nadia Akram has helped in supervision of experimental work and drafting of manuscript.

278 Majid Muneer has helped in supervision of experimental work and drafting of manuscript.

$279 \quad$ Funding

280 Government College University Faisalabad Pakistan provided financial assistance under GCUF281 RSP.

282 Availability of data and materials

283 Available on request

284 References

285 Abdelghany AM, Menazea AA, Abd-El-Maksoud MA, Khatab TK (2020) Pulsed laser ablated 286 zeolite nanoparticles: A novel nano-catalyst for the synthesis of 1,8-dioxo287 octahydroxanthene and N-aryl-1,8-dioxodecahydroacridine with molecular docking 288 validation. Applied Organometallic Chemistry 34:. https://doi.org/10.1002/aoc.5250

289 Achouri F, Corbel S, Balan L, et al (2016) Porous Mn-doped ZnO nanoparticles for enhanced 290 solar and visible light photocatalysis. Materials and Design 101:309-316. 291 https://doi.org/10.1016/j.matdes.2016.04.015

292 Adeleke, J. T., Theivasanthi, T., Thiruppathi, M., Swaminathan, M., Akomolafe, T., \& Alabi AB 293 (2018) Photocatalytic degradation of methylene blue by $\mathrm{ZnO} / \mathrm{NiFe} 2 \mathrm{O} 4$ nanoparticles. 294 Applied Surface Science 455:195-200

295 Alamdari S, Tafreshi MJ, Ghamsari MS (2019) Strong yellow-orange emission from aluminum 
and Indium co-doped $\mathrm{ZnO}$ nanostructures with potential for increasing the color gamut of displays. Applied Physics A: Materials Science and Processing 125:. https://doi.org/10.1007/s00339-019-2451-X

Balcha A, Yadav OP, Dey T (2016) Photocatalytic degradation of methylene blue dye by zinc oxide nanoparticles obtained from precipitation and sol-gel methods. Environmental Science and Pollution Research 23:25485-25493. https://doi.org/10.1007/s11356-016-77506

Bhakta N, Chakrabarti PK (2019) Defect induced room temperature ferromagnetism and optical properties of $(\mathrm{Co}, \mathrm{Y})$ co-doped $\mathrm{ZnO}$ nanoparticles. Journal of Magnetism and Magnetic Materials 485:419-426. https://doi.org/10.1016/j.jmmm.2019.03.106

Cao P, Zhao DX, Shen DZ, et al (2009) Cu + -codoping inducing the room-temperature magnetism and p-type conductivity of $\mathrm{ZnCoO}$ diluted magnetic semiconductor. Applied Surface Science 255:3639-3641. https://doi.org/10.1016/j.apsusc.2008.10.011

Chauhan N, Singh V, Kumar S, et al (2019) Synthesis of nitrogen- and cobalt-doped rod-like mesoporous $\mathrm{ZnO}$ nanostructures to study their photocatalytic activity. Journal of Sol-Gel Science and Technology 91:567-577. https://doi.org/10.1007/s10971-019-05059-3

Dong C, Xiao X, Chen G, et al (2015) Synthesis and photocatalytic degradation of methylene blue over p-n junction Co3O4/ZnO core/shell nanorods. Materials Chemistry and Physics 155:1-8. https://doi.org/10.1016/j.matchemphys.2015.01.033

Fu Z, Pan Z, Sun D, et al (2016) Multiple morphologies of ZnO films synthesized on flexible poly(ethylene terephthalate) by electroless deposition. Materials Letters 184:185-188. https://doi.org/10.1016/j.matlet.2016.08.046 
318

G.H. Kim, D.L. Kim, B.D. Ahn, S.Y. Lee HJK (2009) Investigation on doping behavior of copper in $\mathrm{ZnO}$ thin film. Microelectronics Journal 40:272-275

Hayat H, Mahmood Q, Pervez A, et al (2015) Comparative decolorization of dyes in textile wastewater using biological and chemical treatment. Separation and Purification Technology 154:149-153. https://doi.org/10.1016/j.seppur.2015.09.025

Kanagaraj J, Senthilvelan T, Panda RC (2015) Degradation of azo dyes by laccase: Biological method to reduce pollution load in dye wastewater. Clean Technologies and Environmental Policy 17:1443-1456. https://doi.org/10.1007/s10098-014-0869-6

Kang, K. M., Wang, Y., Kim, M., \& Park HH (2018) Study on properties of Ga/F-co-doped ZnO thin films prepared using atomic layer deposition. Thin Solid Films 660:913-919

Kang KM, Wang Y, Kim M, Park HH (2018) Study on properties of Ga/F-co-doped ZnO thin films prepared using atomic layer deposition. Thin Solid Films 660:913-919. https://doi.org/10.1016/j.tsf.2018.02.030

Kaphle A, Borunda MF, Hari P (2018) Influence of cobalt doping on residual stress in $\mathrm{ZnO}$ nanorods. Materials Science in Semiconductor Processing 84:131-137. https://doi.org/10.1016/j.mssp.2018.05.019

Kaphle A, Reed T, Apblett A, Hari P (2019) Doping efficiency in cobalt-doped ZnO nanostructured materials. Journal of Nanomaterials 2019:. https://doi.org/10.1155/2019/7034620

Katheresan V, Kansedo J, Lau SY (2018) Efficiency of various recent wastewater dye removal methods: A review. Journal of Environmental Chemical Engineering 6:4676-4697. 
https://doi.org/10.1016/j.jece.2018.06.060

Khan I, Sun N, Wang Y, et al (2020) Synthesis of SnO2/yolk-shell LaFeO3 nanocomposites as efficient visible-light photocatalysts for 2,4-dichlorophenol degradation. Materials Research Bulletin 127:. https://doi.org/10.1016/j.materresbull.2020.110857

Khan I, Sun N, Zhang Z, et al (2019) Improved visible-light photoactivities of porous LaFeO3 by coupling with nanosized alkaline earth metal oxides and mechanism insight. Catalysis Science and Technology 9:3149-3157. https://doi.org/10.1039/c9cy00127a

Kumar Y, Sahai A, Olive-Méndez SF, et al (2016) Morphological transformations in cobalt doped zinc oxide nanostructures: Effect of doping concentration. Ceramics International 42:5184-5194. https://doi.org/10.1016/j.ceramint.2015.12.041

Liu N, Li Z (2018) Bimetal-organic frameworks derived carbon doped ZnO/Co3O4 heterojunction as visible-light stabilized photocatalysts. Materials Science in Semiconductor Processing 79:24-31. https://doi.org/10.1016/j.mssp.2018.01.004

Lu JJ, Gu YH, Chen Y, et al (2019) Ultrahigh permeability of graphene-based membranes by adjusting D-spacing with poly (ethylene imine) for the separation of dye wastewater. Separation and Purification Technology 210:737-745. https://doi.org/10.1016/j.seppur.2018.08.065

Ma H, Kong A, Ji Y, et al (2019) Ultrahigh adsorption capacities for anionic and cationic dyes from wastewater using only chitosan. Journal of Cleaner Production 214:89-94. https://doi.org/10.1016/j.jclepro.2018.12.217

Menazea AA, Mostafa AM, Al-Ashkar EA (2020) Impact of CuO doping on the properties of 
$\mathrm{CdO}$ thin films on the catalytic degradation by using pulsed-Laser deposition technique. Optical Materials 100:. https://doi.org/10.1016/j.optmat.2020.109663

Mohamed Reda G, Fan H, Tian H (2017) Room-temperature solid state synthesis of Co3O4/ZnO p-n heterostructure and its photocatalytic activity. Advanced Powder Technology 28:953963. https://doi.org/10.1016/j.apt.2016.12.025

Mohamed RM, McKinney D, Kadi MW, et al (2016) Cobalt/zinc oxide hollow spheres: Visible light nanophotocatalysts. Ceramics International 42:2299-2305. https://doi.org/10.1016/j.ceramint.2015.10.024

Nisar A, Saeed M, Usman M, et al (2020) Kinetic modeling of ZnO-rGO catalyzed degradation of methylene blue. International Journal of Chemical Kinetics 645-654. https://doi.org/10.1002/kin.21389

Noruozi A, Nezamzadeh-Ejhieh A (2020) Preparation, characterization, and investigation of the catalytic property of $\alpha-\mathrm{Fe} 2 \mathrm{O} 3-\mathrm{ZnO}$ nanoparticles in the photodegradation and mineralization of methylene blue. Chemical Physics Letters 752:137587. https://doi.org/10.1016/j.cplett.2020.137587

Pavithra KG, P. SK, Jaikumar V, P. SR (2019) Removal of colorants from wastewater: A review on sources and treatment strategies. Journal of Industrial and Engineering Chemistry 75:119. https://doi.org/10.1016/j.jiec.2019.02.011

Prabakaran E, Pillay K (2019) Synthesis of N-doped ZnO nanoparticles with cabbage morphology as a catalyst for the efficient photocatalytic degradation of methylene blue under UV and visible light. RSC Advances 9:7509-7535. https://doi.org/10.1039/C8RA09962F 
382 Reddy IN, Reddy CV, Shim J, et al (2020) Excellent visible-light driven photocatalyst of (Al,

$383 \mathrm{Ni}$ ) co-doped $\mathrm{ZnO}$ structures for organic dye degradation. Catalysis Today 340:277-285.

$384 \quad$ https://doi.org/10.1016/j.cattod.2018.07.030

385 Saeed M, Adeel S, Ilyas M, et al (2016) Oxidative degradation of Methyl Orange catalyzed by

386 lab prepared nickel hydroxide in aqueous medium. Desalination and Water Treatment

$387 \quad 57: 12804-12813$. https://doi.org/10.1080/19443994.2015.1052992

388 Saeed M, Muneer M, Mumtaz N, et al (2018) Ag-Co3O4: Synthesis, characterization and 389 evaluation of its photo-catalytic activity towards degradation of rhodamine B dye in aqueous medium. Chinese Journal of Chemical Engineering 26:1264-1269. https://doi.org/10.1016/j.cjche.2018.02.024

392 393

Saeed M, Siddique M, Ibrahim M, et al (2020) Calotropis gigantea leaves assisted biosynthesis of $\mathrm{ZnO}$ and $\mathrm{Ag} @ \mathrm{ZnO}$ catalysts for degradation of rhodamine $\mathrm{B}$ dye in aqueous medium. Environmental Progress \& Sustainable Energy. https://doi.org/10.1002/ep.13408

Vuppala V, Motappa MG, Venkata SS, Sadashivaiah PH (2012) Photocatalytic degradation of methylene blue using a zinc oxide-cerium oxide catalyst. European Journal of Chemistry 3:191-195. https://doi.org/10.5155/eurjchem.3.2.191-195.564

Wang CC, Liu M, Man BY, et al (2012) Effect of oxygen pressure on the structural, optical and magnetic properties of pulsed laser ablated $\mathrm{ZnCoO}$ thin films. Journal of Alloys and Compounds 520:158-163. https://doi.org/10.1016/j.jallcom.2011.12.170

Wang W, Yue Q, Li R, et al (2017) Investigating coagulation behavior of chitosan with different Al species dual-coagulants in dye wastewater treatment. Journal of the Taiwan Institute of Chemical Engineers 78:423-430. https://doi.org/10.1016/j.jtice.2017.06.052 
404 Yang Y, Wang X, Yi G, et al (2019) Hydrothermal synthesis of co3 o4/zno hybrid nanoparticles

405 for triethylamine detection. Nanomaterials 9:1-12. https://doi.org/10.3390/nano9111599

406 Zhang D, Su C, Yao S, et al (2020) Facile in situ chemical transformation synthesis, boosted 407 charge separation, and increased photocatalytic activity of BiPO4/BiOCl p-n heterojunction 408 photocatalysts under simulated sunlight irradiation. Journal of Physics and Chemistry of 409 Solids 147:109630. https://doi.org/10.1016/j.jpcs.2020.109630

410 Zhang L, Ye Z, Lu B, et al (2011) Ferromagnetism induced by donor-related defects in Co-doped $411 \quad$ ZnO thin films. Journal of Alloys and Compounds 509:2149-2153.

$412 \quad$ https://doi.org/10.1016/j.jallcom.2010.10.171

413 Zhang W, Camarena DC (2020) Development of ZnFe2O4 nanoparticle functionalized baker's

414 yeast composite for effective removal of methylene blue via adsorption and

415 photodegradation. Journal of Water Process Engineering 37:101234.

416 https://doi.org/10.1016/j.jwpe.2020.101234 